\title{
ON THE ORIGIN OF SPATIALLY DEPENDENT ELECTRONIC EXCITED-STATE DYNAMICS IN MIXED HYBRID PEROVSKITE THIN FILMS*
}

\author{
Y.-Z. Ma ${ }^{\text {a }}$, B. Doughty ${ }^{\text {a }}$, M.J. Simpson ${ }^{\text {a }}$, S. Das ${ }^{\mathrm{b}}$, and K. Xiao ${ }^{\mathrm{c}}$ \\ a Chemical Sciences Division, Oak Ridge National Laboratory, Oak Ridge, Tennessee 37831 \\ ${ }^{\mathrm{b}}$ Department of Electrical Engineering and Computer Science, University of Tennessee, Knoxville, Tennessee 37996 \\ ' Center for Nanophase Materials Sciences, Oak Ridge National Laboratory, Oak Ridge, Tennessee 37831 \\ Email: may1@ornl.gov
}

Received 15 November 2018; accepted 2 January 2019

\begin{abstract}
The fundamental photophysics underlying the remarkable performance of organic-inorganic hybrid perovskites in optoelectronic device applications has been increasingly studied using complementary spectroscopic techniques. However, the spatially heterogeneous polycrystalline morphology of the solution-processed thin films is often overlooked in conventional ensemble measurements and therefore the reported results are averaged over hundreds or even thousands of nano- and micro-crystalline grains. Here, we apply femtosecond transient absorption microscopy to spatially and temporally probe ultrafast electronic excited-state dynamics in chloride containing mixed lead halide perovskite $\left(\mathrm{CH}_{3} \mathrm{NH}_{3} \mathrm{PbI}_{3-x} \mathrm{Cl}_{x}\right)$ thin films. We found that the electronic excited-state relaxation kinetics are extremely sensitive to the spatial location probed, which was manifested by position-dependent transient absorption signal amplitude and decay behaviour, along with an obvious rise component at some positions. The analysis of transient absorption kinetics acquired at several distinct spatial positions enabled us to identify Auger recombination as the dominant mechanism underlying the initial portions of the spatially dependent dynamics with variable rate constants. The different rates observed suggest occurrence of distinct local electronic structures and variable contributions from impurities/defects and phonons in this nonlinear dynamical process.
\end{abstract}

Keywords: transient absorption microscopy, ultrafast electronic excited-state dynamics, Auger recombination, metal halide perovskites

\section{Introduction}

As an emerging solution-processible class of optoelectronic materials, metalhalide perovskites(MHPs) have demonstrated an enormous potential for photovoltaic and light-emitting applications [1-8].

\footnotetext{
* This manuscript has been authored by UT-Battelle, LLC under Contract No. DE-AC05-00OR22725 with the U.S. Department of Energy. The United States Government retains and the publisher, by accepting the article for publication, acknowledges that the United States Government retains a non-exclusive, paid-up, irrevocable, world-wide licence to publish or reproduce the published form of this manuscript, or allow others to do so, for United States Government purposes. The Department of Energy will provide public access to these results of federally sponsored research in accordance with the DOE Public Access Plan https://energy.gov downloads/doe-public-access-plan.
}

The remarkable properties of these materials have stimulated increasingly extensive studies to understand the fundamental photophysics underlying their impressive performance [9-11]. However, the spatial heterogeneity arising largely from the presence of crystalline grains with a varying size, shape, orientation, and level of defects in these thin films [10, 12-16] imposes substantial challenges for such efforts. On the one hand, application of commonly applied ensemble optical spectroscopic techniques to such spatially heterogeneous systems is challenging, as the results obtained will be averaged over many distinct microscopic structures or crystalline grains, which may not represent the intrinsic properties of either the overall material or 
any individual grain within it. In the worst case, the results may even vary with the sample location being probed. On the other hand, to reveal the physical mechanisms underlying the observed spatial dependences of various electronic excitedstate phenomena, an incisive technique with a simultaneous high spatial and temporal resolution, and additionally a capability of assessing the corresponding morphological information is needed. This need has been demonstrated in several timeresolved optical imaging studies based on picosecond photoluminescence (PL) microscopy and femtosecond transient absorption microscopy (TAM). These efforts have enabled the elucidation of several spatially dependent phenomena such as the nature of elementary photoexcitations [17, 18], distributions of photoexcited species [19] and electronic trap states [20, 21], electronic excited-state relaxation [22-24] and transition dipole moment orientations [25], as well as transient sub-bandgap states [26] and charge carrier diffusion [27-29], etc. A combination of steadystate or time-resolved PL microscopy with structural characterization methods such as scanning electron microscopy (SEM) or/and atomic-force microscopy (AFM) has further enabled the correlation of electronic and morphological heterogeneities [22, 23]. Furthermore, a multimodal all-optical imaging approach involving femtosecond TAM, time-integrated PL, transmission, and confocal reflectance modalities developed recently by our laboratory offers an alternative route towards simultaneously probing both electronic and morphological heterogeneities in a contactless and noninvasive manner [30, 31]. Application of this approach to thin films of chloride containing mixed $\mathrm{MHP}\left(\mathrm{CH}_{3} \mathrm{NH}_{3} \mathrm{PbI}_{3-x} \mathrm{Cl}_{x}\right)$ has enabled us to successfully separate bulk and surface contributions to the complex electronic excited-state processes [30].

Despite these exciting advances, it is still not yet well understood why the observed spatial features in the TAM images acquired from high quality thin film samples are often much larger than the corresponding microscopic morphological structures, how and why the underlying electronic dynamics vary from one spatial location to another, and what physical mechanisms govern the associated relaxation dynamics. Here, we report an experimental study probing spatially re- solved ultrafast electronic excited-state dynamics in thin films of chloride containing mixed MHP $\left(\mathrm{CH}_{3} \mathrm{NH}_{3} \mathrm{PbI}_{3-x} \mathrm{Cl}_{x}\right)$ using femtosecond TAM [32]. The key difference between this work and our previous studies [17, 21, 30, 33] on both triiodide and mixed metal halide perovskites lies in a significantly improved spatial resolution - meeting or exceeding the diffraction limit of light. This allows us to examine not only the observed TAM spatial features but also spatially resolved electronic dynamics with a spatial resolution much greater than the microscopic structures of the perovskite grains themselves. The most striking finding of our measurements is that the transient absorption (TA) kinetics extracted from the TAM images are remarkably sensitive to the spatial positions probed in the MHP samples, which is manifested by not only distinct TA signal amplitudes but also different decay kinetics. The analysis of the kinetic data extracted from several spatial locations further allows us to identify an Auger recombination process as the dominant mechanism governing the initial decay dynamics but with clearly different relative rates. These variable Auger rates result from either distinct local electronic structures or different contributions of impurities/defects and phonons to this nonlinear dynamical process or both. Similar to our previous observation, the spatial features seen in the TAM images are again significantly larger in size than the typical grains even with the improved spatial resolution by almost a factor of 2 - we show that the underlying reason for this apparent discrepancy is due to contributions from both emissive and nonemissive species to the TAM signal. The results again underscore the need to resolve electronic excitedstate dynamics of complex materials in both space and time to understand the fundamental photophysics at the heart of light harvesting and emitting technologies.

\section{Experiment}

Femtosecond TAM data was acquired using a modified commercial white light microscope (Eclipse TE 300, Nikon) in combination with a femtosecond laser system as detailed previously [17, 21, 30]. Briefly, a commercial $250 \mathrm{kHz}$ femtosecond Ti:Sapphire regenerative amplifier (RegA 9000, Coherent) and an optical 
parametric amplifier (OPA 9400, Coherent) were employed to produce pump and probe pulses centred at 500 and $750 \mathrm{~nm}$, respectively. The major difference between this work and all our previous optical imaging studies lies in the use of a $100 \times$, 1.40 NA oil immersion objective (Nikon), which offers a significantly improved spatial resolution to image possibly the smallest microscopic structures in the mixed perovskite thin films. TAM images corresponding to a $128 \times 128$ pixel data array were collected by a raster scanning sample stage over a $10 \times 10 \mu \mathrm{m}$ area at a fixed delay time between the pump and probe pulses. The power levels were kept at the lowest possible values while still achieving adequate signal-to-noise ratio: average power levels were approximately $3.5 \mu \mathrm{W}$ pump and $3.2 \mu \mathrm{W}$ probe, corresponding to 14.0 and $12.8 \mathrm{pJ}$ pulse energies, respectively.

The linear absorption spectrum was measured using a Cary $4 \mathrm{E}$ UV-Visible spectrophotometer and a steady-state PL emission spectrum was recorded using a Fluorolog-3 fluorometer with a Hamamatsu R2658 InGaAs photocathode photomultiplier. Bandwidths for the excitation and emission monochromators were 5 and $2 \mathrm{~nm}$, respectively, and a $520 \mathrm{~nm}$ longpass filter was placed in front of the emission monochromator to eliminate residual excitation light scattering.

High quality perovskite films were prepared on a thin \#2 $(\sim 200 \mu \mathrm{m})$ microscope glass cover slip using a previously reported procedure [16]. To protect the film from moisture induced degradation, a thin layer of poly(methyl methacrylate) (PMMA) was spin-coated at $2000 \mathrm{rpm}$ from a 1.5\% PMMA (Sigma-Aldrich) solution in chlorobenzene, as has been used by Savenije and coworkers in their time-resolved spectroscopic experiments [34]. Fresh samples were prepared and kept in a $\mathrm{N}_{2}$-filled glovebox until TAM imaging experiments. The average thickness of the perovskite films was $275 \mathrm{~nm}$, and the RMS roughness was $30-40 \mathrm{~nm}$. The morphology of the samples was examined using a Zeiss Merlin VP scanning electron microscope (SEM).

\section{Results and discussion}

Figure 1 shows the SEM image acquired using a scanning electron microscope (Zeiss Merlin) with $3 \mathrm{KV}$ gun voltage for a typical chloride con-

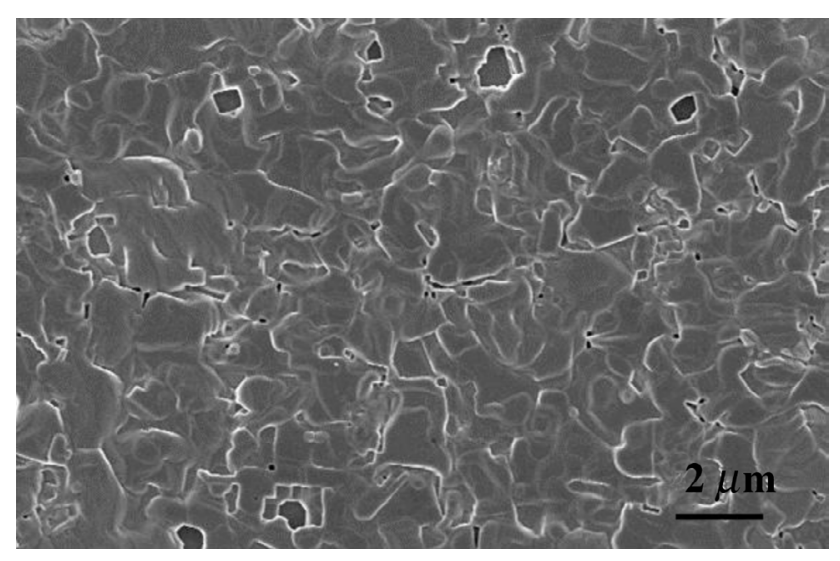

Fig. 1. The SEM image of a chloride containing mixed lead halide perovskite $\left(\mathrm{CH}_{3} \mathrm{NH}_{3} \mathrm{PbI}_{3-x} \mathrm{Cl}_{x}\right)$ film. The scale bar is $2 \mu \mathrm{m}$.

taining a mixed $\mathrm{MHP}\left(\mathrm{CH}_{3} \mathrm{NH}_{3} \mathrm{PbI}_{3-x} \mathrm{Cl}_{x}\right)$ thin film, which was prepared following the same procedure as those used for our TAM imaging measurements described in this paper, but without a protective PMMA layer. From this image, one can clearly see distinct grains with a typical size on the order of 1-2 $\mu \mathrm{m}$. In addition, the presence of only very few pinholes in the thin film further shows a high quality of our sample, in contrast to those used in some of recent reports based on femtosecond TAM measurements.

The typical spectra of linear absorption and steady-state PL emission measured for the thin film samples used for our TAM imaging experiments are shown in Fig. 2. Based on the positions

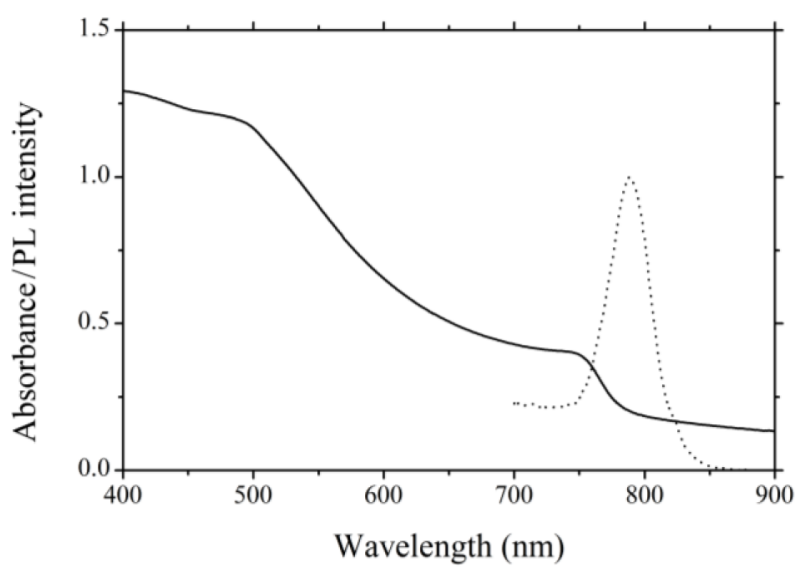

Fig. 2. Linear absorption (a solid line) and steadystate PL emission (a dash line) spectra of a chloride containing mixed lead halide perovskite $\left(\mathrm{CH}_{3} \mathrm{NH}_{3} \mathrm{PbI}_{3-x} \mathrm{Cl}_{x}\right)$ film sample. The PLemission spectrum was measured upon an excitation at $500 \mathrm{~nm}$. 
of the absorption edge and the PL emission band, we expect the TA signal detected with a probe pulse centred at $750 \mathrm{~nm}$ and a bandwidth of $25 \mathrm{~nm}$ (fullwidth at half maximum) should be dominated by photoinduced bleaching and stimulated emission as indeed observed from the TAM measurements detailed below.

The representative TAM images collected at five delay times are shown in Fig. 3. From these images, one can immediately identify several striking features. First, the amplitudes of the observed TA signals depend strongly on the spatial positions probed in the film, but the TA signal sign remains unchanged across the entire image. The same TA signal sign indicates that the dominant contributions are from both photoinduced bleaching and stimulated emission. Second, the observed spatial features evolve temporally on distinct timescales, which can be seen by comparing the images collected at different delay times. Given the heterogeneous morphology due to the presence of crystalline grains with a variable size, shape and orientation as shown in Fig. 1, as well as a possibly distinct level of defects from one position to another, it is not surprising that the TA kinetics are so sensitive to the spatial position within this material. Third, the observed spatial features are significantly larger than the typical grain sizes, which are on the order of 1-2 $\mu \mathrm{m}$ as shown in Fig. 1. The observed spatial features are in fact very similar to those seen in our previous TAM measurements on mixed $\mathrm{CH}_{3} \mathrm{NH}_{3} \mathrm{PbI}_{3-x} \mathrm{Cl}_{x}$ perovskite thin films using the same microscope and laser wavelengths but a different objective with a comparably lower NA (0.75) [30]. They are, however, noticeably smaller than those seen in triiodide $\mathrm{CH}_{3} \mathrm{NH}_{3} \mathrm{PbI}_{3}$ perovskite thin films [17], which were again acquired using the same imaging settings as described in Ref. [30] except that the probe wavelength was centred at $800 \mathrm{~nm}$. Different sizes in the observed spatial features may reflect relatively larger grains in the triiodide perovskite films than the ones in the mixed perovskites. Despite the difference in the sizes of the observed spatial features, a common observation for both triiodide and mixed perovskite samples is the absence of any observable grain boundaries in the acquired TAM images. Furthermore, these observed spatial features in the TAM images are again comparable in size to those seen in one- and two-photon fluorescence imaging studies [35]. Given the high spatial resolu-

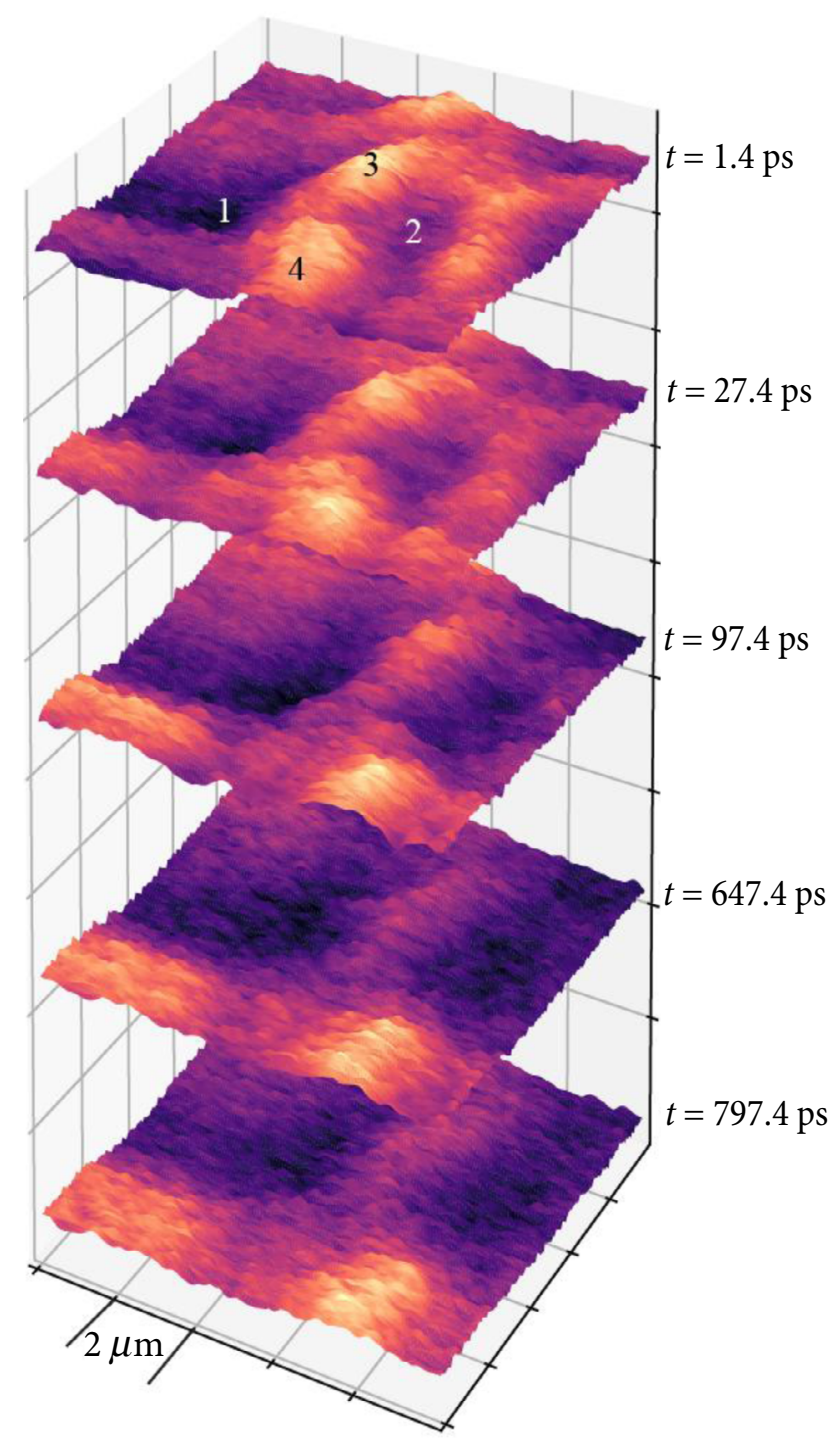

Fig. 3. TAM images acquired for the same area of a $\mathrm{CH}_{3} \mathrm{NH}_{3} \mathrm{PbI}_{3-x} \mathrm{Cl}_{x}$ thin film sample at five different delay times as indicated. Scale bars are $2 \mu \mathrm{m}$, and the image size is $10 \times 10 \mu \mathrm{m}$, corresponding to $128 \times 128$ pixels. ROI \#1, 2 and 3 correspond to the spatial locations with low, medium and high TA signal amplitudes, respectively, and ROI \#4 depicts a region with a clear rise in the TA kinetics.

tion afforded by the higher NA objective used in this work, absence of any resolvable grain boundaries here is somewhat unexpected. Since much abundant spatial features with sizes comparable to the grains can be readily observed with PL imaging even with a lower NA objective [21, 30], the clearly larger TAM features seen here and previously are obviously not the result of an insufficient spatial resolution. Also, the surface- and bulk-specificities of these imaging modalities for strongly absorbing 
MHP thin films [30] cannot account for the remarkably distinct spatial features observed either, simply because some of the grains are in fact as tall as the film thickness [12] and therefore should be resolvable in both modalities. In view of the different photoexcited species being probed by PL and TA spectroscopies, emissive species in the former whereas both emissive and nonemissive in the latter, we believe that the absence of observable grain boundaries in the TAM images arises from nonemissive species contribution to the TA signal at the grain boundaries. Thus, the results in Fig. 3 show that the overall photoexcitation distribution in such highly heterogeneous systems must occur on a larger length scale than the spatial heterogeneity seen in the thin film morphology.

The remarkable dependence of the observed TA signals on the sample spatial location probed can be more clearly seen by directly comparing the kinetic traces obtained by integrating over smaller $1.0 \times 1.0 \mu \mathrm{m}$ areas at selected positions of the TAM images shown in Fig. 3 and plotting those signals as a function of the delay time between the pump and probe pulses. Figure 4 shows the kinetics obtained from three different positions of the TAM images with low, medium and high signal amplitudes as indicated on the TAM images shown in Fig. 3, corresponding to the region of interest (ROI) labelled as 1,2 and 3, respectively. The results of their deconvolution fitting are also included in Fig. 3, and all the fitting parameters are summarized in Table 1. Besides the apparent differences in the peak signal amplitudes, the overall relaxation timescales differ as well, which is manifested by an accelerated decay with increasing peak amplitude. As the TA signal amplitude at a given delay time is proportional to $\left(\sigma_{\mathrm{PA}}-\sigma_{\mathrm{PB}}-\sigma_{\mathrm{SE}}\right) n(t)$, where $\sigma_{\mathrm{PA}}, \sigma_{\mathrm{PB}}$ and $\sigma_{\mathrm{SE}}$ are the cross sections of photoinduced absorption (PA), stimulated emission (SE) and photoinduced bleaching (PB), respectively, and $n(t)$ is the charge carrier density [36], the observed differences may arise from either spatially dependent $\sigma_{\mathrm{PA}}, \sigma_{\mathrm{PB}}, \sigma_{\mathrm{SE}^{\prime}}$ and $n(t)$, or a combination of them. To distinguish these possibilities, we first consider the slower decay behaviour of the TA kinetics by scaling the data to an approximately equal amplitude at long delay times. As shown in Fig. 4(d), this slower decay behaviour appears to be very similar within the experimental uncertainty, and therefore the observed differences must be dominated by the fast relaxation

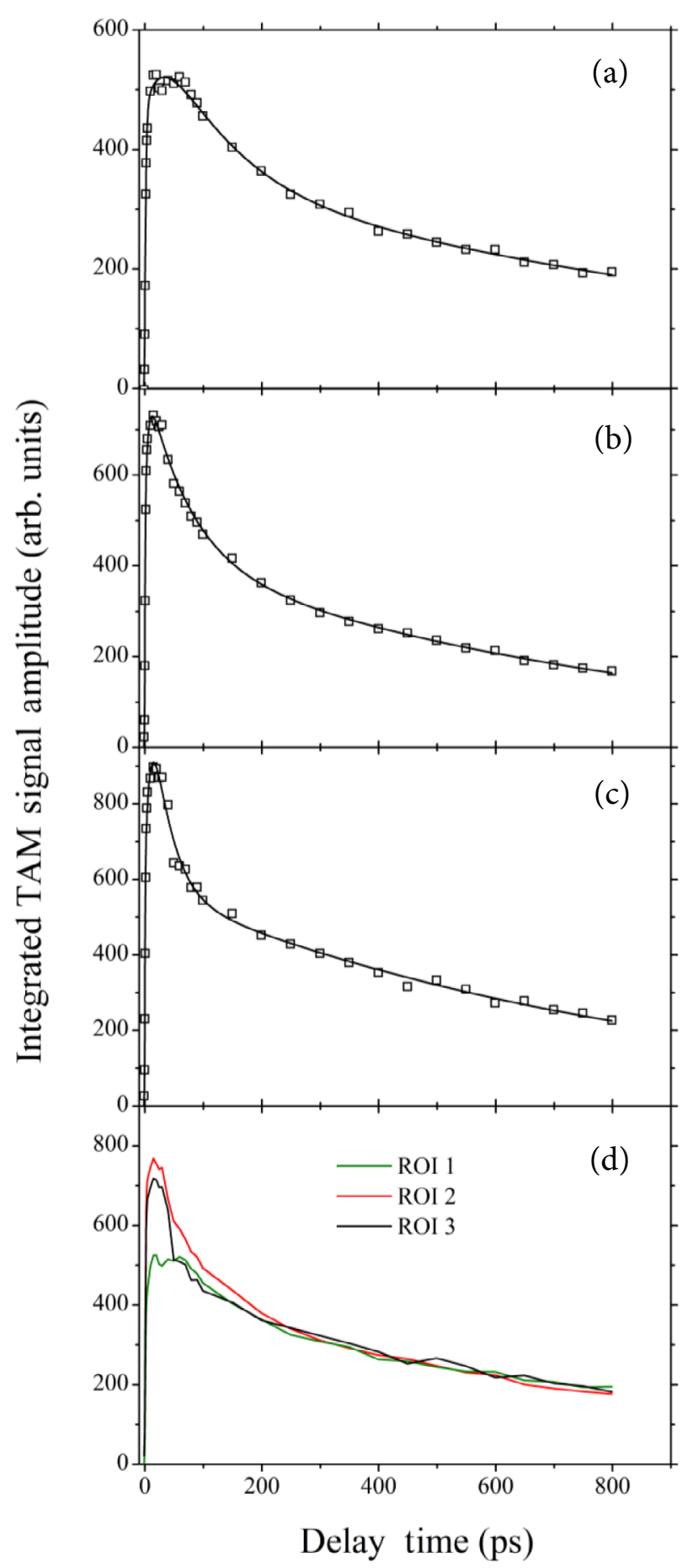

Fig. 4. TA kinetics at three different locations of the TAM image with low (a), medium (b) and high (c) signal amplitude features. The precise locations correspond to the ROIs labelled as 1, 2 and 3 in the TAM images shown in Fig. 3. These kinetic traces were obtained by integrating a $1.0 \times 1.0 \mu \mathrm{m}$ image area, and the solid lines are the results of deconvolution fitting. Same kinetic traces scaled to an approximately equal amplitude at long delay times (d). 
Table 1. Summary of the time constants $\left(\tau_{1}-\tau_{4}\right)$ and relative amplitudes $\left(a_{1}-a_{4}\right)$ determined for the TA kinetics obtained by integrating different regions of interest (ROI) and the entire image area.

\begin{tabular}{c|c|c|c|c|c|c|c|c}
\hline ROI & $\tau_{1}, \mathrm{ps}$ & $a_{1}, \%$ & $\tau_{2}, \mathrm{ps}$ & $a_{2}, \%$ & $\tau_{3}, \mathrm{ps}$ & $a_{3}, \%$ & $\tau_{4}, \mathrm{ps}$ & $a_{4}, \%$ \\
\hline 1 & 1.9 & -57.9 & 31.5 & -32.5 & 113 & 48.1 & Const. & 51.9 \\
\hline 2 & 4.7 & -38.8 & - & - & 73.6 & 48.8 & Const. & 51.2 \\
\hline 3 & 8.9 & -53.5 & 31.5 & 57.0 & - & - & Const. & 43.0 \\
\hline 4 & 2.9 & -13.7 & 48.0 & -68.3 & 67.5 & 69.2 & Const. & 30.8 \\
\hline Total & 2.8 & -34.9 & 25.9 & -17.3 & 99.6 & 42.2 & Const. & 57.8 \\
\hline
\end{tabular}

A long-lived decay component whose time constant cannot be determined due to the relatively short scan range is specified as 'Const.' in the Table.

dynamics within the first 200 ps. Next, we explore the physical mechanism underlying the observed kinetic differences. Although the pump power employed for acquiring the TAM images is about four times lower than that used in our previous TAM experiment on triiodide $\mathrm{CH}_{3} \mathrm{NH}_{3} \mathrm{PbI}_{3}$ thin films [17], use of different microscope objectives in current (1.40 NA) and previous ( $0.72 \mathrm{NA}$ ) experiments will lead to an approximately four times smaller pump focal spot in this work than that in our previous study. This would lead to a comparable pump fluence in the current and previous experiments. As a result, we expect that the nonlinear carrier dynamics such as Auger recombination observed previously will also play a dominant role here. As such nonlinear dynamical processes are very sensitive to the initial density of photoinduced charge carriers, the observed differences may be explained by assuming generation of distinct initial densities of charge carriers at different spatial locations without invoking spatially dependent cross sections. Occurrence of these distinct carrier densities may result from spatially dependent sample absorption owing to primarily different orientations and heights of grains. A higher carrier density at a given spatial location will not only lead to a larger TA signal amplitude but also a more pronounced charge carrier relaxation given that the effect induced by Auger recombination is proportional to the third power of the carrier density, which would in turn result in a more rapid decay in the early timescale. This expectation is fully consistent with the experimental results shown in Fig. 4 .

An even more dramatic spatial dependence of kinetics is found at the ROI \#4 in the TAM images in Fig. 3. As shown in Fig. 5 and also Table 1, a satisfactory description of this kinetic trace would not be possible without inclusion of a slower rise com- ponent with a timescale of 48 ps. The subsequent relaxation process appears to be clearly slower than any of the kinetics shown in Fig. 4. Although the exact cause for this unique kinetic behaviour is unclear, we speculate that rapid charge carrier trapping results in limited carrier migration and associated interactions. For comparison, we further show the kinetic trace obtained by integrating the entire $10 \times 10 \mu \mathrm{m}$ image area in Fig. 5(b). Clearly, the decay behaviour is substantially different from either of the kinetic traces obtained by integrating

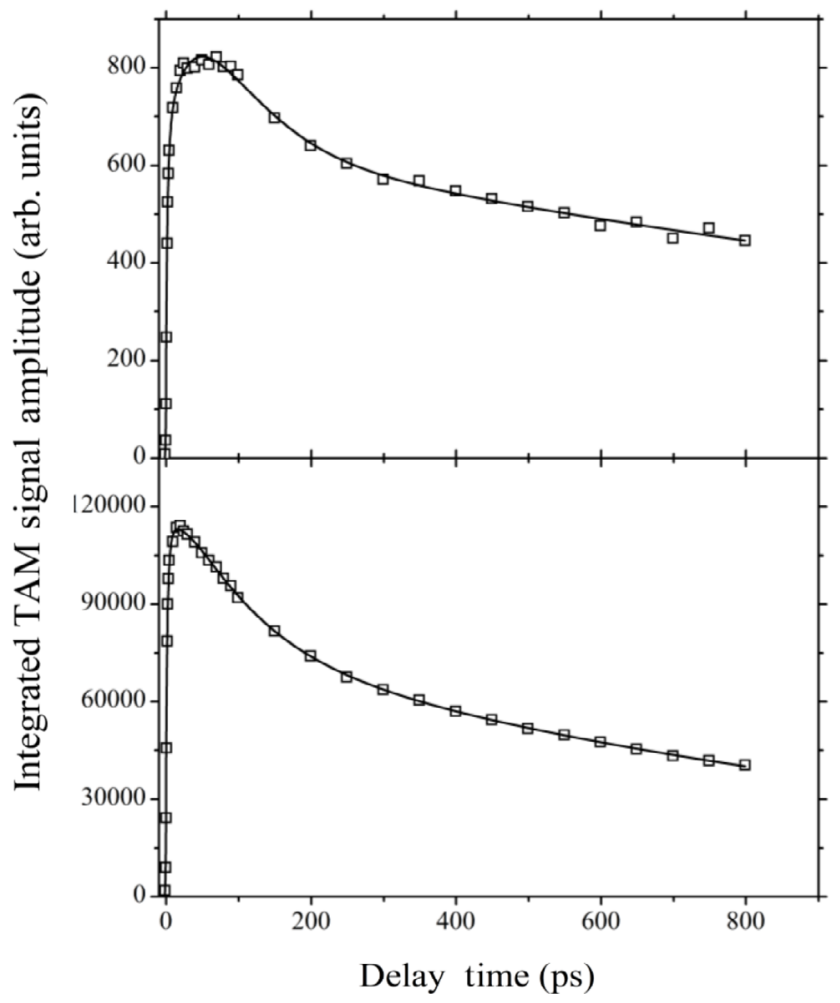

Fig. 5. TA kinetics obtained by integrating a small $1.0 \times 1.0 \mu \mathrm{m}$ area (a) at a chosen location labelled as ROI \#4 in the TAM image shown in Fig. 3, and the entire $10 \times 10 \mu \mathrm{m}$ image area (b). The solid lines are the results of deconvolution fitting. 
a smaller area at all four selected ROIs as shown in Figs. 4 and 5. As ensemble TA measurements integrate the entire spatial overlapping region of the pump and probe beam spots, which have typical diameters ranging from tens to hundreds of $\mathrm{mi}-$ crometres, the results will describe an average over hundreds or even thousands of individual crystalline grains. It is therefore challenging to capture the electronic excited-state dynamics associated with well-defined crystalline grains of a micrometre size using more commonly employed ensemble spectroscopic measurements. Unless the sampling area is sufficiently large, the data collected using ensemble measurements might even vary with the sample position or/and the changes in the spot size of the pump and probe beams. This further highlights the critical need for a simultaneous high spatial and temporal resolution to unambiguously reveal both ultrafast excited-state dynamics and underlying physical mechanisms in such spatially heterogeneous systems.

Further insight about the spatially dependent kinetics can be obtained by explicitly considering the Auger recombination of charged carriers [37, 38] at early delay times. According to the analysis described in detail in Refs. [39, 40], the equation describing this nonlinear dynamical process can be written as

$$
\frac{1}{[n(t)]^{2}}-1=\gamma_{\mathrm{A}} t,
$$

where $n(t)$ is the charge carrier density at delay time, $t$, and $\gamma_{\mathrm{A}}$ is the rate constant of Auger recombination. Note this equation holds only for early delay times when the nonlinear dynamical process dominates the linear relaxation. As the TA signal, $\Delta O D(t)$, is proportional to $n(t)$, this equation offers a straightforward means to qualitatively compare the Auger recombination rates at distinct spatial locations by simply plotting the squared inverse of the TA signal amplitude, $1 /[\Delta O D(t)]^{2}$, as a function of the delay time, $t$. As one can see from Eq. (1), the slopes of the linear portions of these plots are directly proportional to the Auger recombination rate constants.

As shown in Fig. 6, the linear dependence of 1/ $[\Delta O D(t)]^{2}$ on $t$ is clearly evident in the early delay times for all the kinetics shown in Figs. 4 and 5, but the corresponding slopes are clearly different. Specifically, the slope reduces in the order of

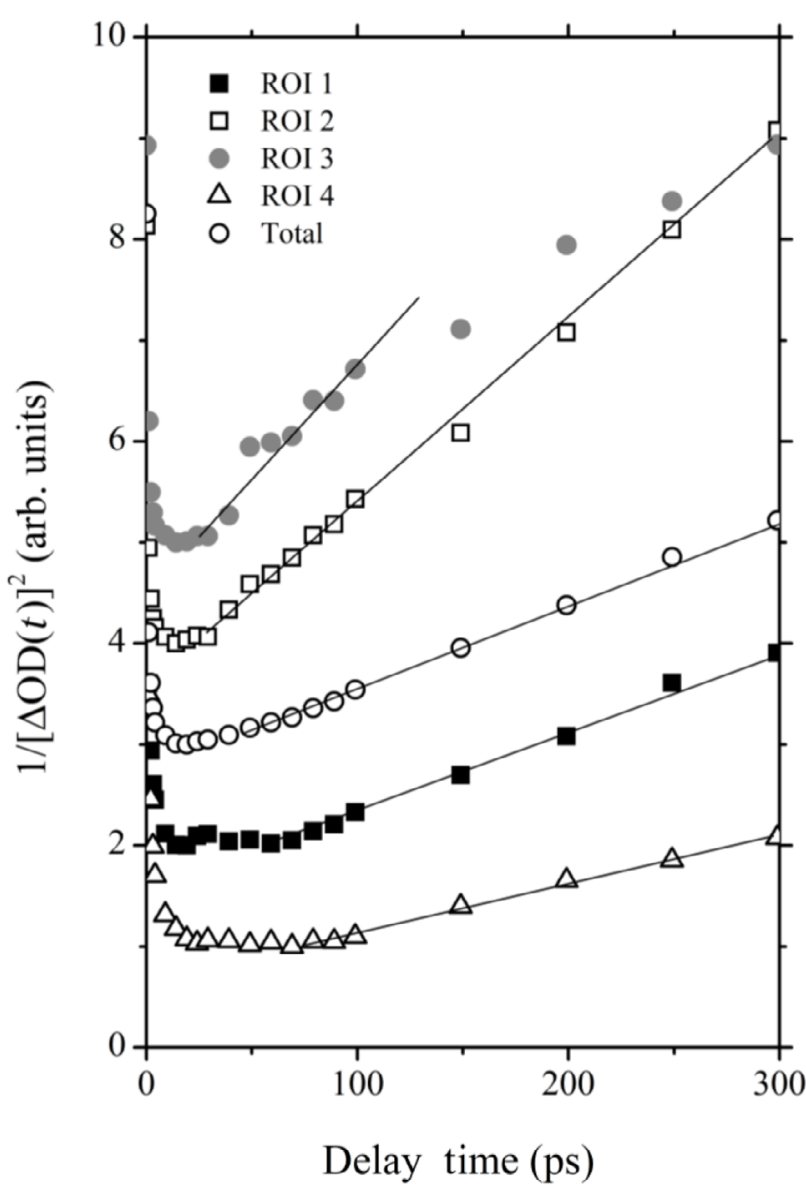

Fig. 6. The plot of $1 /[\Delta O D(t)]^{2}$ vs delay time for the data obtained at the ROI \#1, 2, 3 and 4 in the TAM images shown in Fig. 3. The result for the entire image is also shown for completeness. Note that the peak amplitudes of all the kinetic data were normalized to 1.0 prior to calculating the squared inverses, and the solid lines are drawn to guide the eye. The calculated squared inverses are vertically offset for clarity.

decreasing the TA signal amplitude for the spatial features with the high, medium and low amplitude. The slope for the TA kinetics obtained by the integrated entire image lies between those associated with the medium and low amplitude features, whereas the spatial feature at the ROI \#4 has the smallest slope due to its significantly slower decay than all other kinetics. This analysis shows that the Auger recombination rate depends on the spatial location in this heterogeneous system, and these distinct rates also contribute to the observed spatially dependent kinetics at early delay times as shown in Figs. 4 and 5 . Consequently, the observed spatially dependent electronic excited-state dynamics is expected to originate from the creation of spatially distinct charge 
carrier densities in combination with their resulting Auger recombination with variable rates. As the Auger recombination rate in semiconductors depends strongly on the electronic band structure [41] and can be further affected by impurities and phonons [42], the different rates observed in this work may arise from the presence of distinct local grain structures or/and a variable involvement of impurities/defects and phonons in this nonlinear dynamical process. For solution-processed polycrystalline thin films, all these variations should be possible, and, in fact, all these influences on the Auger recombination rates have been indeed experimentally observed in various triiodide and mixed MHPs [43-45].

Although we found that an Auger recombination process plays a dominant role in the initial portions of the spatially resolved electronic excited-state dynamics, which lasts approximately 200 ps as shown in Fig. $4(\mathrm{~d})$, the distinct spatial features observed in the TAM images are by no means the result of this nonlinear electronic relaxation. Instead, they describe an intrinsic heterogeneity of charge carrier distribution in the polycrystalline MHP thin films, which is clearly evident by comparing the TAM images acquired at long and short delay times such as 1.4 and 797.4 ps, respectively. The seemingly 'blurred' images at long delay times result from charge carrier diffusion within the thin films. Taking the diffusion coefficients of $0.042 \pm 0.016 \mathrm{~cm}^{2} \mathrm{~s}^{-1}$ for electrons and $0.054 \pm 0.022 \mathrm{~cm}^{2} \mathrm{~s}^{-1}$ for holes [46], we can estimate that these charge carriers should diffuse 116 and $132 \mathrm{~nm}$, respectively, from their initial positions of photogeneration after $800 \mathrm{ps}$. As a result, the probe pulse will see a decreased carrier density, leading to a low TA signal and image contrast.

In conclusion, femtosecond TAM was applied to study ultrafast electronic excited-state dynamics in spatially heterogeneous chloride containing mixed lead halide perovskite $\left(\mathrm{CH}_{3} \mathrm{NH}_{3} \mathrm{PbI}_{3-x} \mathrm{Cl}_{x}\right)$ thin films. In comparison to our previous studies on both triiodide and mixed metal halide perovskite thin films, the results presented here are obtained with an improved spatial resolution by employing a microscope objective with higher NA while maintaining adequate time resolutions to clock excited state dynamics with a sufficiently high temporal resolution. However, we found that achieving a higher spatial resolution does not allow one to resolve finer spa- tial structures than those previously observed, and this finding was attributed to the contributions from both emissive and nonemissive photoexcited species to TA signals. Our most striking finding is that the observed TA kinetics are extremely sensitive to the spatial position probed, manifested by distinct TA signal amplitudes in combination with different decay/rise signatures. The analysis of the decay behaviour of the kinetics acquired from spatially distinct locations enabled us to identify the Auger recombination as the dominant mechanism underlying the initial spatially dependent kinetics with variable rate constants. Based on these observations, we conclude that the observed spatially dependent electronic excited-state dynamics originates from a combination of creation of spatially distinct charge carrier densities and their resulting Auger recombination with variable rates. The remarkable difference between the TA kinetic traces obtained by integrating the entire TA images and distinct subimages further demonstrates the need for a novel spectroscopic technique with a simultaneous spatial and temporal resolution in order to unambiguously determine the excited-state relaxation processes and underlying physical mechanisms in such systems with a high spatial heterogeneity. We thus expect that these and future ultrafast nonlinear imaging studies will provide a greater insight into the fundamental electronic processes underlying optoelectronic devices based on this class of novel materials.

\section{Acknowledgements}

Work by M.J.S., B.D. and Y.-Z.M. was supported by the U.S. Department of Energy, Office of Science, Basic Energy Sciences, Chemical Sciences, Geosciences, and Biosciences Division. The perovskite sample preparation and SEM characterization by S.D. and K.X. was conducted at the Center for Nanophase Materials Sciences (CNMS), which is a DOE Office of Science User Facility.

\section{References}

[1] S.D. Stranks and H.J. Snaith, Metal-halide perovskites for photovoltaic and light-emitting devices, Nat. Nanotech. 10, 391-402 (2015), https:// doi.org/10.1038/nnano.2015.90

[2] Q. Lin, A. Armin, P.L. Burn, and P. Meredith, Organohalide perovskites for solar energy 
conversion, Acc. Chem. Res. 49, 545-553 (2016), https://doi.org/10.1021/acs.accounts.5b00483

[3] B.R. Sutherland and E.H. Sargent, Perovskite photonic sources, Nat. Photon. 10, 295-302 (2016), https://doi.org/10.1038/nphoton.2016.62

[4] T.M. Brenner, D.A. Egger, L. Kronik, G. Hodes, and D. Cahen, Hybrid organic-inorganic perovskites: low-cost semiconductors with intriguing charge-transport properties, Nat. Rev. Mater. 1, 15007 (2016), https://doi.org/10.1038/natrevmats.2015.7

[5] J.-P. Correa-Baena, A. Abate, M. Saliba, W. Tress, T. Jesper Jacobsson, M. Grätzel, and A. Hagfeldt, The rapid evolution of highly efficient perovskite solar cells, Energy Environ. Sci. 10, 710-727 (2017), https://doi.org/10.1039/C6EE03397K

[6] M. Saliba, J.-P. Correa-Baena, M. Grätzel, A. Hagfeldt, and A. Abate, Perovskite solar cells: from the atomic level to film quality and device performance, Angew. Chem., Int. Ed. 57, 2554-2569 (2018), https://doi.org/10.1002/anie.201703226

[7] J. Huang, Y. Shao, and Q. Dong, Organometal trihalide perovskite single crystals: a next wave of materials for $25 \%$ efficiency photovoltaics and applications beyond? J. Phys. Chem. Lett. 6, 3218-3227 (2015), https://doi.org/10.1021/acs. jpclett.5b01419

[8] N.-G. Park, M. Grätzel, T. Miyasaka, K. Zhu, and K. Emery, Towards stable and commercially available perovskite solar cells, Nat. Energy 1, 16152 (2016), https://doi.org/10.1038/nenergy.2016.152

[9] T.C. Sum and N. Mathews, Advancements in perovskite solar cells: photophysics behind the photovoltaics, Energy Environ. Sci. 7, 2518-2534 (2014), https://doi.org/10.1039/C4EE00673A

[10]J.A. Christians, J.S. Manser, and P.V. Kamat, Multifaceted excited state of $\mathrm{CH}_{3} \mathrm{NH}_{3} \mathrm{PbI}_{3}$. Charge separation, recombination, and trapping, J. Phys. Chem. Lett. 6, 2086-2095 (2015), https:// doi.org/10.1021/acs.jpclett.5b00594

[11]A.R.S. Kandada and A. Petrozza, Photophysics of hybrid lead halide perovskites: The role of microstructure, Acc. Chem. Res. 49, 536-544 (2016), https://doi.org/10.1021/acs.accounts.5b00464

[12]B. Yang, O. Dyck, J. Poplawsky, J. Keum, A. Puretzky, S. Das, I. Ivanov, C. Rouleau, G. Duscher,
D. Geohegan, and K. Xiao, Perovskite solar cells with near $100 \%$ internal quantum efficiency based on large single crystalline grains and vertical bulk heterojunctions, J. Am. Chem. Soc. 137, 9210-9213 (2015), https://doi.org/10.1021 jacs.5b03144

[13]H. Zhou, Q. Chen, G. Li, S. Luo, T.-B. Song, H.-S. Duan, Z. Hong, J. You, Y. Liu, and Y. Yang, Interface engineering of highly efficient perovskite solar cells, Science 345, 542-546 (2014), https://doi.org/10.1126/science.1254050

[14]W. Nie, H. Tsai, R. Asadpour, J.-C. Blancon, A.J. Neukirch, G. Gupta, J.J. Crochet, M. Chhowalla, S. Tretiak, M.A. Alam, H.-L. Wang, and A.D. Mohite, High-efficiency solution-processed perovskite solar cells with millimeter-scale grains, Science 347, 522-525 (2015), https://doi. org/10.1126/science.aaa0472

[15]X. Wu, M.T. Trinh, D. Niesner, H. Zhu, Z. Norman, J.S. Owen, O. Yaffe, B.J. Kudisch, and X.-Y. Zhu, Trap states in lead iodide perovskites, J. Am. Chem. Soc. 137, 2089-2096 (2015), https://doi.org/10.1021/ja512833n

[16]S. Das, G. Gu, P.C. Joshi, B. Yang, T. Aytug, C.M. Rouleau, D.B. Geohegan, and K. Xiao, Low thermal budget, photonic-cured compact $\mathrm{TiO}_{2}$ layers for high-efficiency perovskite solar cells, J. Mater. Chem. A 4, 9685-9690 (2016), https:// doi.org/10.1039/C6TA02105K

[17]M.J. Simpson, B. Doughty, B. Yang, K. Xiao, and Y.-Z. Ma, Spatial localization of excitons and charge carriers in hybrid perovskite thin films, J. Phys. Chem. Lett. 6, 3041-3047 (2015), https:// doi.org/10.1021/acs.jpclett.5b01050

[18]S. Nah, B. Spokoyny, C. Stoumpos, C.M.M. Soe, M. Kanatzidis, and E. Harel, Spatially segregated free-carrier and exciton populations in individual lead halide perovskite grains, Nat. Photon. 11, 285 (2017), https://doi.org/10.1038/nphoton.2017.36

[19]M.J. Simpson, B. Doughty, B. Yang, K. Xiao, and Y.-Z. Ma, Separation of distinct photoexcitation species in femtosecond transient absorption microscopy, ACS Photonics 3, 434-442 (2016), https://doi.org/10.1021/acsphotonics.5b00638

[20]S. Draguta, S. Thakur, Y. Morozov, Y. Wang, J.S. Manser, P.V. Kamat, and M. Kuno, Spatially 
non-uniform trap state densities in solution-processed hybrid perovskite thin films, J. Phys. Chem. Lett. 7, 715-721 (2016), https://doi.org/10.1021 acs.jpclett.5b02888

[21]M.J. Simpson, B. Doughty, B. Yang, K. Xiao, and Y.-Z. Ma, Imaging electronic trap states in perovskite thin films with combined fluorescence and femtosecond transient absorption microscopy, J. Phys. Chem. Lett. 7, 1725-1731 (2016), https://doi.org/10.1021/acs.jpclett.6b00715

[22]D.W. deQuilettes, S.M. Vorpahl, S.D. Stranks, H. Nagaoka, G.E. Eperon, M.E. Ziffer, H.J. Snaith, and D.S. Ginger, Impact of microstructure on local carrier lifetime in perovskite solar cells, Science 348, 683-686 (2015), https://doi. org/10.1126/science.aaa5333

[23] M. Vrućinić, C. Matthiesen, A. Sadhanala, G. Divitini, S. Cacovich, S.E. Dutton, C. Ducati, M. Atatüre, H. Snaith, R.H. Friend, H. Sirringhaus, and F. Deschler, Local versus long-range diffusion effects of photoexcited states on radiative recombination in organic-inorganic lead halide perovskites, Adv. Sci. 1500136 (2015), https://doi. org/10.1002/advs.201500136

[24]C. Schnedermann, J.M. Lim, T. Wende, A.S. Duarte, L. Ni, Q. Gu, A. Sadhanala, A. Rao, and P. Kukura, Sub-10 femtosecond time-resolved vibronic microscopy, J. Phys. Chem. Lett. 7, 4854-4859 (2016), https://doi.org/10.1021/acs. ipclett.6b02387

[25] B.R. Watson, B. Yang, K. Xiao, Y.-Z. Ma, B. Doughty, and T.R. Calhoun, Elucidation of perovskite film micro-orientations using two-photon total internal reflectance fluorescence microscopy, J. Phys. Chem. Lett. 6, 3283-3288 (2015), https://doi.org/10.1021/acs.jpclett.5b01474

[26]S. Nah, B. Spokoyny, X. Jiang, C. Stoumpos, C.M.M. Soe, M.G. Kanatzidis, and E. Harel, Transient sub-bandgap states in halide perovskite thin films, Nano Lett. 18, 827-831 (2018), https://doi.org/10.1021/acs.nanolett.7b04078

[27]Z. Guo, J.S. Manser, Y. Wan, P.V. Kamat, and L. Huang, Spatial and temporal imaging of longrange charge transport in perovskite thin films by ultrafast microscopy, Nat. Commun. 6, 7471 (2015), https://doi.org/10.1038/ncomms8471
[28]Z. Guo, Y. Wan, M. Yang, J. Snaider, K. Zhu, and L. Huang, Long-range hot-carrier transport in hybrid perovskites visualized by ultrafast microscopy, Science 356, 59-62 (2017), https://doi. org/10.1126/science.aam7744

[29]Z. Guo, N. Zhou, O.F. Williams, J. Hu, W. You, and A.M. Moran, Imaging carrier diffusion in perovskites with a diffractive optic-based transient absorption microscope, J. Phys. Chem. C 122, 10650-10656 (2018), https://doi.org/10.1021/acs. ipcc.8b03643

[30]M.J. Simpson, B. Doughty, S. Das, K. Xiao, and Y.-Z. Ma, Separating bulk and surface contributions to electronic excited-state processes in hybrid mixed perovskite thin films via multimodal all-optical imaging, J. Phys. Chem. Lett. 8, 3299-3305 (2017), https://doi.org/10.1021/acs. ipclett.7b01368

[31]B.J. Foley, S. Cuthriell, S. Yazdi, A.Z. Chen, S.M. Guthrie, X. Deng, G. Giri, S.-H. Lee, K. Xiao, B. Doughty, Y.-Z. Ma, and J.J. Choi, Impact of crystallographic orientation disorders on electronic heterogeneities in metal halide perovskite thin films, Nano Lett. 18, 6271-6278 (2018), https://doi.org/10.1021/acs. nanolett.8b02417

[32]W. Min, C.W. Freudiger, S. Lu, and X.S. Xie, Coherent nonlinear optical imaging: beyond fluorescence microscopy, Annu. Rev. Phys. Chem. 62, 507-530 (2011), https://doi.org/10.1146/annurev. physchem.012809.103512

[33]B. Doughty, M.J. Simpson, B. Yang, K. Xiao, and Y.-Z. Ma, Simplification of femtosecond transient absorption microscopy data from $\mathrm{CH}_{3} \mathrm{NH}_{3} \mathrm{PbI}_{3}$ perovskite thin films into decay associated amplitude maps, Nanotechnology 27, 114002 (2016), https://doi.org/10.1088/0957-4484/27/11/114002

[34]E.M. Hutter, G.E. Eperon, S.D. Stranks, and T.J. Savenije, Charge carriers in planar and meso-structured organic-inorganic perovskites: mobilities, lifetimes, and concentrations of trap states, J. Phys. Chem. Lett. 6, 3082-3090 (2015), https://doi.org/10.1021/acs.jpclett.5b01361

[35] X.M. Wen, R. Sheng, A.W.Y. Ho-Baillie, A. Benda, S. Woo, Q.S. Ma, S.J. Huang, and M.A. Green, Morphology and carrier extraction study of 
organic-inorganic metal halide perovskite by one- and two-photon fluorescence microscopy, J. Phys. Chem. Lett. 5, 3849-3853 (2014), https:// doi.org/10.1021/jz502014r

[36]Y.-Z. Ma, C.D. Spataru, L. Valkunas, S.G. Louie, and G.R. Fleming, Spectroscopy of zigzag singlewalled carbon nanotubes: comparing femtosecond transient absorption spectra with $a b$ initio calculations, Phys. Rev. B 74, 085402 (2006), https://doi.org/10.1103/PhysRevB.74.085402

[37]M. Ghanassi, M.C. Schanne-Klein, F. Hache, A.I. Ekimov, D. Ricard, and C. Flytzanis, Timeresolved measurements of carrier recombination in experimental semiconductor-doped glasses: Confirmation of the role of Auger recombination, Appl. Phys. Lett. 62, 78-80 (1993), https:// doi.org/10.1063/1.108833

[38] V.I. Klimov, A.A. Mikhailovsky, D.W. McBranch, C.A. Leatherdale, and M.G. Bawendi, Quantization of multiparticle Auger rates in semiconductor quantum dots, Science 287, 1011-1013 (2000), https://doi.org/10.1126/science.287.5455.1011

[39]H. Htoon, J.A. Hollingsworth, R. Dickerson, and V.I. Klimov, Effect of zero- to one-dimensional transformation on multiparticle Auger recombination in semiconductor quantum rods, Phys. Rev. Lett. 91, 227401 (2003), https://doi. org/10.1103/PhysRevLett.91.227401

[40]Y.-Z. Ma, L. Valkunas, S.M. Bachilo, and G.R. Fleming, Exciton binding energy in semiconducting single-walled carbon nanotubes,
J. Phys. Chem. B 109, 15671-15674 (2005), https://doi.org/10.1021/jp053011

[41]A. Haug, Auger recombination in direct-gap semiconductors: band-structure effects, J. Phys. C 16, 4159 (1983), https://doi.org/10.1088/00223719/16/21/017

[42] M. Takeshima, Unified theory of the impurity and phonon scattering effects on Auger recombination in semiconductors, Phys. Rev. B 25, 5390-5414 (1982), https://doi.org/10.1103/PhysRevB.25.5390

[43]L.M. Herz, Charge-carrier dynamics in organic-inorganic metal halide perovskites, Annu. Rev. Phys. Chem. 67, 65-89 (2016), https://doi. org/10.1146/annurev-physchem-040215-112222

[44] R.L. Milot, G.E. Eperon, H.J. Snaith, M.B. Johnston, and L.M. Herz, Temperature-dependent charge-carrier dynamics in $\mathrm{CH}_{3} \mathrm{NH}_{3} \mathrm{PbI}_{3}$ perovskite thin films, Adv. Funct. Mater. 25, 6218-6227 (2015), https://doi.org/10.1002/adfm.201502340

[45]W. Rehman, R.L. Milot, G.E. Eperon, C. Wehrenfennig, J.L. Boland, H.J. Snaith, M.B. Johnston, and L.M. Herz, Charge-carrier dynamics and mobilities in formamidinium lead mixed-halide perovskites, Adv. Mater. 27, 7938-7944 (2015), https://doi.org/10.1002/adma.201502969

[46]S.D. Stranks, G.E. Eperon, G. Grancini, C. Menelaou, M.J.P. Alcocer, T. Leijtens, L.M. Herz, A. Petrozza, and H.J. Snaith, Electron-hole diffusion lengths exceeding 1 micrometer in an organometal trihalide perovskite absorber, Science 342, 341-344 (2013), https://doi.org/10.1126/science.1243982

\title{
DĖL ERDVIŠKAI PRIKLAUSOMŲ ELEKTRONINIŲ SUŽADINTŲ BŪSENŲ DINAMIKOS MIŠRIŲJŲ HIBRIDINIŲ PEROVSKITŲ PLONUOSIUOSE SLUOKSNIUOSE KILMĖS
}

\author{
Y.-Z. Ma a , B. Doughty a , M.J. Simpson ${ }^{\text {a }}$, S. Das ${ }^{\text {b }}$, K. Xiao a \\ ${ }^{a}$ Ouk Ridžo nacionalinè laboratorija, Ouk Ridžas, Tenesis \\ ${ }^{\mathrm{b}}$ Tenesio universitetas, Noksvilis, Tenesis
}

